\section{OC-064 NATURAL HISTORY OF UNEXPLAINED BILIARY DILATION}

doi:10.1136/gut.2011.239301.64 bile duct and what follow-up was carried out. Patients with unexplained biliary dilation were followed via search of radiological database and clinical record database.

Results 1386 MRCP results were reviewed from May 2005 to July 2009. 348 (25.1\%) were identified in which a dilated bile duct was reported but no obstructing lesion was seen. $17(4.9 \%)$ patients were excluded because they were referred from an outside institution for MRCP and follow-up data was unavailable, leaving 331 for review. Of these, presumed diagnoses for biliary dilatation included gallstones $(n=146$, $44.1 \%)$, post-cholecystectomy $(\mathrm{n}=86,26 \%)$, pancreatitis $(\mathrm{n}=$ $27,8.1 \%)$, cholecystitis ( $\mathrm{n}=17,5.1 \%)$, duodenal diverticulum $(\mathrm{n}=4,1.2 \%)$, choledochocele $(\mathrm{n}=1,0.3 \%)$, gallbladder surgery unspecified $(n=1,0.3 \%)$ and strawberry gallbladder $(n=$ $1,0.3 \%)$. There were $48(14.5 \%)$ cases of unexplained biliary dilation, which constituted the follow-up group. Females predominated $(n=31,64.6 \%$ ). Mean age was 65 years (range 34-96). Duct diameter ranged from 7-22 mm (mean 11 $\mathrm{mm})$. Follow-up was available on 44 patients (91.6\%). Mean follow-up was 35 months (range 6-64 months). 8 (18.2\%) patients had ERCP. All were normal except 1 (12.5\%), demonstrating choledocholithiasis. 9 (20.5\%) patients had duodenoscopy, all of which were normal. One patient (2.3\%) had an endoscopic ultrasound, which was normal. Mortality in the follow-up group was $13.6 \%$ ( $n=6 ; 1$ myocardial infarction, 1 respiratory failure, 1 uterine carcinoma, 1 intracranial haemorrhage, 1 unknown, 1 pancreatic carcinoma). Two (4.5\%) patients were diagnosed with carcinoma head of pancreas 14 months and 8 months, respectively, after their index MRCP. One was an 87-year-old female who had an ERCP, which confirmed duct dilation but no obstructive cause. A third CT scan 14 months later confirmed probable metastatic carcinoma from head of pancreas. The other was a 34-year-old man diagnosed with carcinoma head of pancreas 8 months later after presenting with obstructive jaundice followed by an ERCP and stent. He died 21 months after his index MRCP.

Conclusion In this retrospective study of incidental unexplained biliary dilatation, a small but significant $4.5 \%$ of patients were diagnosed with a malignant aetiology. We recommend that all patients with incidental unexplained duct dilation on MRCP are closely followed up and considered for endoscopic ultrasound.

Competing interests None.

Keywords ampullary carcinoma, bile duct, cholangiocarcinoma, endoscopic retrograde cholangiopancreatography, endoscopic ultrasound, magnetic resonance imaging, pancreatic cancer.

Introduction With the increasing use and availability of MRCP imaging incidental duct dilation is common. There are no guidelines as to how these patients should be investigated or followed. We sought to retrospectively identify a cohort of patients with biliary dilation on MRCP and assess the incidence of sinister pathology.

Methods Local MRCP database was searched to identify patients with biliary dilation. Data was collected on patient demographics, radiological diagnosis, diameter of common 